\title{
Factores que influyen en la madre, en el abandono de la lactancia
}

\author{
Factors that influence the mother, in the abandonment of breastfeeding \\ Mirna Patricia Valle Rosas, ${ }^{*}$ Osvaldo García Torres, ${ }^{\ddagger}$ Andrea Socorro Álvarez Villaseñor ${ }^{\S}$
}

\section{RESUMEN}

Introducción: De acuerdo con la OMS, la lactancia materna exclusiva (LME) consiste en dar al lactante únicamente leche materna: no otros líquidos ni sólidos, ni siquiera agua, excepto soluciones de rehidratación. Aun cuando se conocen los beneficios al lactante por parte de la madre, existe abandono de ésta. Objetivo: Conocer los principales factores que influyen en la madre para el abandono de la lactancia. Material y métodos: Estudio observacional, transversal, descriptivo a 90 madres derechohabientes, en el HGZ +MF No. 1 en La Paz, Baja California Sur. Se realizó, previa firma de consentimiento informado, la encuesta de Niño y colaboradores que mide factores asociados al abandono precoz de la lactancia materna con 20 ítems agrupados en variables sociodemográficos maternos y sociodependientes. Se utilizó estadística descriptiva para variables demográficas y $\chi^{2}$ para asociación; todas las participantes firmaron consentimiento informado. Resultados: El 48.4\% son madres trabajadoras. Los factores maternos asociados al abandono de la LME fueron: el inicio de actividades de las madres (9.9\%), enfermedad del niño (8.8\%), que el lactante no queda satisfecho, queda con hambre (6.6\%), enfermedad de la madre con utilización de fármacos excretados por leche materna (5.5\%), rechazo del niño al pecho (4.4\%), falta de leche (4.4\%), otros motivos (14.3\%). Conclusión: Los factores maternos asociados al abandono de la LME que influyeron en este estudio fueron inicio de actividades de la madre, enfermedad del lactante, que el niño no queda satisfecho, queda con hambre.

Palabras clave: Abandono de la lactancia materna, factores asociados.

\begin{abstract}
Introduction: According to $\mathrm{WHO}$, exclusive breastfeeding consists of giving the infant only breast milk: no other liquids or solids, not even water, except rehydration solutions. Even when the benefits to the nursing mother are known, there is abandonment of the mother. Objective: To know the main factors that influences the mother, for the abandonment of breastfeeding. Material and methods: Observational, cross-sectional, descriptive study of 90 eligible mothers, in the HCZ +MF No. 1 in La Paz, Baja California Sur. The survey by Niño et al is carried out after signing the informed consent, which measures factors associated with the early abandonment of breastfeeding with 20 items grouped into maternal and socio-dependent socio-demographic variables. Descriptive statistics were used for demographic variables and $\chi^{2}$ for association; all participants signed informed consent. Results: $48.4 \%$ are working mothers. The maternal factors associated with the abandonment of EB were: the initiation of activities of the mothers (9.9\%), illness of the child (8.8\%), that the infant was not satisfied, left hungry (6.6\%), illness of the mother with use of drugs excreted through breast milk (5.5\%), rejection of the child to the breast (4.4\%), lack of milk (4.4\%), other reasons (14.3\%). Conclusion: The maternal factors associated with the abandonment of EBF that influenced this study were the initiation of activities of the mother, illness of the infant that the child is not satisfied, is left hungry.
\end{abstract}

Keywords: Abandonment of breastfeeding, associated factors.

* Médico familiar. Unidad de Medicina Familiar No. 14.

₹ Profesor titular del curso de especialización en medicina familiar. Hospital General de Zona +MF No. 1.

${ }^{\S}$ Coordinación auxiliar médica de investigación. Instituto Mexicano del Seguro Social.

La Paz, Baja California Sur, México.

Correspondencia: ASAV, andrea.alvarez@imss. gob.mx

Conflicto de intereses:

Ninguno.

Citar como: Valle RMP,

García TO, Álvarez VAS.

Factores que influyen en la madre, en el abandono de la lactancia. Rev CONAMED. 2020; 25(4): 167-173. https:// dx.doi.org/10.35366/97336 Financiamiento: No se contó con financiamiento externo.

Recibido: 20/02/2020. Aceptado: 07/12/2020 


\section{INTRODUCCIÓN}

La lactancia materna (LM) se considera un factor de suma importancia entre la alimentación de un niño y el apego emocional entre la madre y éste. La Organización Mundial de la Salud (OMS) recomienda la lactancia materna exclusiva durante los primeros seis meses de vida del niño, para que éste logre un desarrollo óptimo; así como recibir alimentos complementarios, sin abandonar por completo la lactancia materna hasta los dos años de edad del infante?

La UNICEF lanza un comunicado de prensa en 2013 en el que menciona: «No hay ninguna otra

Tabla 1: Características generales de las madres encuestadas. $\mathrm{N}=90$.

\begin{tabular}{|c|c|c|}
\hline Variable & & n (\%) \\
\hline \multirow[t]{3}{*}{ Ocupación } & Ama de casa & $41(45.0)$ \\
\hline & Trabajadora & $43(48.4)$ \\
\hline & Estudiante & $6(6.6)$ \\
\hline \multirow[t]{6}{*}{ Nivel educativo } & Básica incompleta & $3(3.3)$ \\
\hline & Básica completa & 19 (20.9) \\
\hline & Media incompleta & $8(8.8)$ \\
\hline & Media completa & $32(35.2)$ \\
\hline & Universitaria incompleta & $7(7.7)$ \\
\hline & Universitaria completa & $21(24.2)$ \\
\hline \multirow[t]{6}{*}{ *Nivel socioeconómico } & Alta & $9(9.9)$ \\
\hline & Media alta & $23(25.3)$ \\
\hline & Media & $12(13.2)$ \\
\hline & Media baja & $24(27.5)$ \\
\hline & Baja alta & $11(12.1)$ \\
\hline & Baja & $11(12.1)$ \\
\hline \multirow[t]{4}{*}{ Estado civil } & Soltera & 19 (20.9) \\
\hline & Casada & $40(45.1)$ \\
\hline & Conviviente & $30(33.0)$ \\
\hline & Sin pareja & $1(1.1)$ \\
\hline \multirow[t]{5}{*}{ Número total de hijos } & 1 & $40(44.0)$ \\
\hline & 2 & $33(37.4)$ \\
\hline & 3 & $15(16.5)$ \\
\hline & 4 & $1(1.1)$ \\
\hline & 5 & $1(1.1)$ \\
\hline \multirow[t]{2}{*}{ Orientación previa de lactancia materna } & Sí & $82(90.1)$ \\
\hline & No & $8(9.9)$ \\
\hline \multirow[t]{2}{*}{ Cuándo recibió orientación de lactancia materna } & Antes del embarazo & $1(1.1)$ \\
\hline & Durante el embarazo & 89 (98.9) \\
\hline \multirow[t]{2}{*}{ Abandono de lactancia materna exclusiva } & Sí & $48(53.3)$ \\
\hline & No & $42(46.2)$ \\
\hline
\end{tabular}


intervención de salud que tenga mayores repercusiones para los bebés y las madres que la lactancia materna, y que cueste menos a los gobiernos». A través de la Directora Ejecutiva Adjunta de la UNICEF, Geeta Rao Gupta, se emitió el siguiente mensaje: "La lactancia materna exclusiva es la 'primera inmunización' de un bebé, y la forma más eficaz y barata de salvar vidas». ${ }^{2}$

Buenaño y colegas identificaron que el dejar a los hijos periodos largos sin poder lactar es el principal factor psicosocial que influye en el abandono de la lactancia materna exclusiva (LME), debido a que deben incorporarse a sus actividades diarias laborales, lo que causa que la madre introduzca alimentación con sucedáneos. ${ }^{3}$

Campiño y su grupo identifican tres principales causas de abandono de lactancia materna entre las que se encuentran producción insuficiente de leche referida por la madre 30\%, el niño rechazaba el seno $13 \%$ y la madre debía ingresar a trabajar 9\%.4,5

Otros factores de abandono de lactancia descritos por Acosta y su equipo son el comienzo de los estudios, tareas laborales y por estética, ${ }^{6}$ además que incorporarse a la vida laboral es necesario para contribuir al sustento económico familiar y este factor influye en que se complemente la lactancia con sucedáneos. ${ }^{?}$

Debido a que los factores de abandono de la lactancia materna pueden presentar una frecuencia diferente de acuerdo a la población que se estudia, el objetivo de la presente investigación es identificar los factores maternos asociados al abandono de la lactancia materna exclusiva.

\section{MATERIAL Y MÉTODOS}

Estudio observacional, de tipo encuesta transversal, descriptivo, en 90 mujeres con hijo de más de seis meses a un año de edad, de un universo de 1,273 adscritas al HCZ +MF No. 1 La Paz, Baja California Sur, en el periodo de 2018-2019, que acudieron a consulta externa de medicina familiar para identificar los factores maternos asociados al abandono de la lactancia materna exclusiva. El tamaño de la muestra se realizó mediante la fórmula de una sola proporción y un muestreo no probabilístico de casos consecutivos con una frecuencia esperada del evento de $6 \%$ de abandono en madres trabajadoras.
Tabla 2: Motivo de abandono de lactancia materna.

\begin{tabular}{lc}
\hline Ítem & $\mathrm{n}(\%)$ \\
\hline Continua con lactancia & $42(46.2)$ \\
Inicio de actividades & $9(9.9)$ \\
Enfermedad del niño & $8(8.8)$ \\
No queda satisfecho, queda con hambre & $6(6.6)$ \\
Enfermedad de la madre con utilización de & $5(5.5)$ \\
fármacos excretados por leche materna & \\
Falta de leche & $4(4.4)$ \\
Rechazo del niño al pecho & $4(4.4)$ \\
*Otros & $3(4.4)$ \\
Enfermedad de la madre relacionada con & $3(3.3)$ \\
la mama o el pezón & \\
Incorporación a la sala de cuna & $2(2.2)$ \\
Voluntariedad de la madre & $2(2.2)$ \\
Estética & $2(2.2)$ \\
\hline
\end{tabular}

* No especificados.

Se eliminaron aquellas encuestas incompletas. Se utilizó el cuestionario de lactancia materna de Niño y colaboradores, que evalúan los factores relacionados al abandono de la lactancia exclusiva; las características del encuestado como la edad, estado civil, ocupación, nivel educativo, número total de hijos, orientación previa de lactancia materna, cuándo recibió orientación de lactancia materna (antes del embarazo o durante el embarazo); principales fuentes de información, sexo del lactante, vía de nacimiento, experiencia de lactancia previa, duración de lactancia exclusiva, ablactación, motivo de abandono de lactancia materna.

El nivel socioeconómico se evaluó a través de NSE-8X7 (A/B: alta; C+: media alta; C: media; C-: media baja; D+: baja alta; y D: baja). Previa firma de consentimiento informado a la madre o tutor del lactante mayor de seis meses.

Para el análisis de variables numéricas y de razón se utilizó un análisis descriptivo con medidas de tendencia central (media) y dispersión (media, desviación estándar). Para las variables cualitativas (dicotómicas y categóricas) se utilizaron frecuencias (\%). En el análisis inferencial de caso de las variables evaluadas mediante frecuencias se utilizó 
la $\chi^{2}$ de Pearson, con cálculo de intervalos de confianza al 95\%. Un valor de p igual o menor a 0.05 fue considerado como significativo.

Una vez recolectados los datos se procedió a codificar las respuestas para efectuar el procesamiento de los datos y el análisis estadístico pertinente, usando el paquete Excel 2010 y SPSS 21.

El estudio es considerado sin riesgo según la ley general de salud vigente en México, y fue autorizado por el comité de investigación y ética en investigación de la unidad hospitalaria con el registro R-2018-301-017.

\section{RESULTADOS}

Se realizó un estudio observacional, transversal, descriptivo en 90 mujeres con hijo de más de seis meses a un año de edad adscritas al Hospital General de Zona con Medicina Familiar No. 1 de La Paz, Baja California Sur, para identificar los factores maternos asociados al abandono de la lactancia materna exclusiva.

La media de edad global fue de $28.29 \pm 6.069$ años, un rango de 26 años, como mínimo de 16 años y máximo de 42 años. Cuarenta y tres mujeres (48.4\%)

Tabla 3: Motivo de abandono de lactancia materna vs ocupación. $\mathrm{N}=90$.

\begin{tabular}{|c|c|c|c|c|}
\hline \multirow[b]{2}{*}{ *Factor de abandono } & \multicolumn{4}{|c|}{ Ocupación } \\
\hline & & $\begin{array}{l}\text { Ama de casa } \\
\qquad(\mathrm{n}, \%)\end{array}$ & $\begin{array}{l}\text { Trabajadora } \\
\qquad(n, \%)\end{array}$ & $\begin{array}{l}\text { Estudiante } \\
\qquad(\mathrm{n}, \%)\end{array}$ \\
\hline Enfermedad del niño & $\begin{array}{l}\text { Sí } \\
\text { No }\end{array}$ & $\begin{array}{c}3(3.3) \\
38(42.2)\end{array}$ & $\begin{array}{c}\mathbf{5} \text { (5.5) } \\
39(43.3)\end{array}$ & $\begin{array}{l}0(0.0) \\
5(5.5)\end{array}$ \\
\hline Estética & $\begin{array}{l}\text { Sí } \\
\text { No }\end{array}$ & $\begin{array}{c}1(1.1) \\
40(44.4)\end{array}$ & $\begin{array}{c}0(0.0) \\
44(46.6)\end{array}$ & $\begin{array}{l}1(1.1) \\
4(4.4)\end{array}$ \\
\hline *Otros & $\begin{array}{l}\text { Sí } \\
\text { No }\end{array}$ & $\begin{array}{c}2(2.2) \\
39(43.3)\end{array}$ & $\begin{array}{c}2(2.2) \\
42(95.4)\end{array}$ & $\begin{array}{l}0(0.0) \\
5(5.5)\end{array}$ \\
\hline Continua con lactancia & $\begin{array}{l}\text { Sí } \\
\text { No }\end{array}$ & $\begin{array}{r}20(22.2) \\
21(23.3)\end{array}$ & $\begin{array}{l}19(21.1) \\
25(22.2)\end{array}$ & $\begin{array}{l}3(3.3) \\
2(2.2)\end{array}$ \\
\hline $\begin{array}{l}\text { Enfermedad de la madre con utilización de } \\
\text { fármacos excretados por leche materna }\end{array}$ & $\begin{array}{l}\text { Sí } \\
\text { No }\end{array}$ & $\begin{array}{c}1(1.1) \\
40(44.4)\end{array}$ & $\begin{array}{c}3(3.3) \\
44(48.8)\end{array}$ & $\begin{array}{l}1(1.1) \\
1(1.1)\end{array}$ \\
\hline $\begin{array}{l}\text { Enfermedad de la madre relacionada con la } \\
\text { mama o el pezón }\end{array}$ & $\begin{array}{l}\text { Sí } \\
\text { No }\end{array}$ & $\begin{array}{c}3(3.3) \\
38(42.2)\end{array}$ & $\begin{array}{c}0(0.0) \\
44(48.8)\end{array}$ & $\begin{array}{l}0(0.0) \\
5(5.5)\end{array}$ \\
\hline Rechazo del niño al pecho & $\begin{array}{l}\text { Sí } \\
\text { No }\end{array}$ & $\begin{array}{l}4(4.4) \\
37(41.1)\end{array}$ & $\begin{array}{c}0(0.0) \\
44(48.8)\end{array}$ & $\begin{array}{l}0(0.0) \\
5(5.5)\end{array}$ \\
\hline No queda satisfecho, queda con hambre & $\begin{array}{l}\text { Sí } \\
\text { No }\end{array}$ & $\begin{array}{c}2(2.2) \\
39(95.1)\end{array}$ & $\begin{array}{c}4(\mathbf{4 . 4 )} \\
40(90.0)\end{array}$ & $\begin{array}{l}0(0.0) \\
5(100)\end{array}$ \\
\hline Incorporación a la sala de cuna & $\begin{array}{l}\text { Sí } \\
\text { No }\end{array}$ & $\begin{array}{c}0(0.0) \\
41(45.5)\end{array}$ & $\begin{array}{c}2(\mathbf{2 . 2}) \\
42(46.6)\end{array}$ & $\begin{array}{l}0(0.0) \\
5(5.5)\end{array}$ \\
\hline Falta de leche & $\begin{array}{l}\text { Sí } \\
\text { No }\end{array}$ & $\begin{array}{c}2(2.2) \\
39(43.3)\end{array}$ & $\begin{array}{c}2(2.2) \\
42(46.6)\end{array}$ & $\begin{array}{l}0(0.0) \\
5(5.5)\end{array}$ \\
\hline Voluntariedad de la madre & $\begin{array}{l}\text { Sí } \\
\text { No }\end{array}$ & $\begin{array}{c}1(1.1) \\
40(44.4)\end{array}$ & $\begin{array}{c}1(1.1) \\
43(47.7)\end{array}$ & $\begin{array}{l}0(0.0) \\
5(5.5)\end{array}$ \\
\hline Inicio de actividades & $\begin{array}{l}\text { Sí } \\
\text { No }\end{array}$ & $\begin{array}{c}2(2.2) \\
39(43.3)\end{array}$ & $\begin{array}{r}6(6.6) \\
37(41.7)\end{array}$ & $\begin{array}{l}1(1.1) \\
5(5.5)\end{array}$ \\
\hline
\end{tabular}

\footnotetext{
* Cuestionario de factores de abandono.
} 
Tabla 4: Correlación de variables de abandono de lactancia materna exclusiva.

\begin{tabular}{|c|c|c|c|c|}
\hline \multirow[b]{2}{*}{ Variable } & & \multicolumn{2}{|c|}{$\begin{array}{l}\text { Abandono de lactancia } \\
\text { materna exclusiva }\end{array}$} & \multirow[b]{2}{*}{$\begin{array}{c}{ }^{*} p \\
\text { IC }(95 \%)\end{array}$} \\
\hline & & $\begin{array}{c}\text { Sí }<6 \text { meses } \\
(n, \%)\end{array}$ & $\begin{array}{c}\text { No }>6 \text { meses } \\
(n, \%)\end{array}$ & \\
\hline \multirow[t]{4}{*}{ Experiencia de lactancia previa } & Buena & 15 (16.6) & $21(23.3)$ & 0.011 \\
\hline & Regular & $9(10.0)$ & $0(0.0)$ & $(0.007-0.010)$ \\
\hline & Mala & $2(2.2)$ & $2(2.2)$ & \\
\hline & ‡No aplica & $22(24.4)$ & $19(21.0)$ & \\
\hline \multirow[t]{10}{*}{ Mes de ablactación } & 1 & $0(0.0)$ & $1(1.1)$ & 0.072 \\
\hline & 3 & $1(1.1)$ & $0(0.0)$ & (0.0.23-0.029) \\
\hline & 4 & $8(8.8)$ & $0(0.0)$ & \\
\hline & 5 & $3(3.3)$ & $0(0.0)$ & \\
\hline & 6 & $24(26.4)$ & $31(34.4)$ & \\
\hline & 7 & $2(2.2)$ & $1(1.1)$ & \\
\hline & 8 & $2(2.2)$ & $3(3.3)$ & \\
\hline & 9 & $6(6.6)$ & $5(5.5)$ & \\
\hline & 10 & $2(1.1)$ & $0(0.0)$ & \\
\hline & 12 & $0(0.0)$ & $1(1.1)$ & \\
\hline
\end{tabular}

${ }^{*} \chi^{2} ; \ddagger$ Primípara.

son trabajadoras, 32 de las encuestadas tienen nivel educativo de media completa (35.2\%), predominó en 23 el nivel socioeconómico medio alto (25.3\%), 40 mujeres refirieron estar casadas (45.1\%), 40 son madres primerizas (44\%), 82 tuvieron orientación previa de lactancia (90.1\%), 48 mujeres abandonaron la lactancia (53.3\%) y ocho (9.9\%) no recibió orientación previa de lactancia materna (Tabla 7).

Dentro de los factores de abandono de la lactancia predominó el inicio de actividades en nueve (9.9\%), tal como se describe en la Tabla 2.

De las 42 mujeres (46.2\%) que continuaron con lactancia materna exclusiva más de seis meses, las características de éstas fueron las siguientes: madre casada, 18 (19.8\%); madre conviviente, 15 (16.5\%); seguida de madre soltera en nueve casos (9.9\%). Doce mujeres tuvieron nivel educativo universitario completo (13.2\%) y 12 (13.2\%) media superior completa.

De acuerdo a la ocupación de la madre, predominó el abandono en la mujer trabajadora, 25 (60.9\%); y los factores más comunes de abandono fueron los siguientes: enfermedad de la madre con utilización de fármacos excretados por leche materna, tres (3.3\%); no queda satisfecho, queda con hambre, cuatro (4.4\%); incorporación a la sala de cuna, dos (2.2\%); e inicio de actividades, seis (6.6\%) (Tabla 3).

Al hacer el análisis inferencial del tiempo de abandono de lactancia (< de seis meses), se encontró asociación significativa con la experiencia de la madre en lactancia previa ( $p=0.011$; IC 95\% 0.007-0.010) (Tabla 4).

\section{DISCUSIÓN}

En el presente estudio realizado en 90 mujeres, existe una media de 28.29 años de edad perteneciente a la tercera década de la vida, semejante a la reportada por González Huéscar y su equipo, con una diferencia de dos años menos en la media (26.8 años). Se tuvo una semejanza entre mujeres trabajadoras y dedicadas al hogar y un $6.6 \%$ de estudiantes, las cuales son tres veces a las reportadas por González, quien reporta una 
proporción dos a una de las dedicadas al hogar (catalogada como desempleadas) a las trabajadoras, por lo que se infiere que existe un cambio laboral entre los estudios. ${ }^{8}$

En este estudio se encontró que los motivos de abandono de la lactancia materna son multifactoriales: predominó el inicio de actividades de la madre, en 9.9\%; 8.8\% por enfermedad del niño; 5.5\% por enfermedad de la madre con la utilización de fármacos excretados por la leche materna; $4.4 \%$ otros y 2.2\% estética. Lo anterior se puede comparar con lo reflejado por Prado y colegas donde 78\% refirió presentar dificultades para dar de lactar. De las madres que sí presentan dificultades, 87.1\% refirió disminución de leche y $5.1 \%$ mala formación del pezón. ${ }^{9}$

Con respecto a la orientación previa, 83.6\% de las pacientes refirieron haber recibido algún tipo de orientación previa de lactancia materna, 16.4\% refiere no haber recibido orientación previa sobre lactancia materna, paralelo a lo que se comenta en el estudio de Acosta Silva y su grupo, el nivel de conocimiento de la población estudiada fue bajo en $76.71 \%$ y adecuado sólo en $23.29 \%$. $^{6}$

La principal fuente de información sobre el tema fue el personal de servicios de salud (42.9\%), por parte del personal de enfermería, seguido de la información otorgada por el médico (27.5\%), la familia (19.8\%) y finalmente medios masivos de comunicación (1.1\%). En comparación a lo reportado por Martínez y colaboradores: 59\% de las madres encuestadas obtuvo información acerca de la lactancia materna de su familia, 38\% obtuvo información del personal de salud y 3\% obtuvo información de sus amigos. ${ }^{10}$

En lo referente al resultado de la vía del nacimiento fue en $39.6 \%$ vía vaginal y $60.4 \%$ vía cesárea, datos muy similares con lo que reporta Reyes y su equipo, donde reportan que 57\% fue mediante cesárea y 43\% fue por vía vaginal."

En relación a la duración de la lactancia materna resultó que $12.1 \%$ de las madres abandonaron la lactancia materna exclusiva en el primer mes de vida del lactante y $34.4 \%$ a los seis meses posterior al nacimiento. Mínguez y equipo, en 2014, describieron que la incidencia de abandono de la lactancia materna exclusiva en el primero, segundo y cuarto mes postparto fue de 53.6, 47.6 y 69.6\%, respectivamente. ${ }^{12}$
En este estudio el principal motivo del abandono de la lactancia materna fue debido al inicio de actividades; a diferencia de lo presentado por Cardoña, quien encontró que 29.6\% de las madres abandonaron la lactancia antes de los seis meses, con mayor frecuencia en 83.8\% por deficiencia en la producción de leche, seguido de $22.1 \%$ rechazo de la lactancia por parte del lactante y en $17.6 \%$ cumplimiento de horario de trabajo. De acuerdo con Macay Regina la incorporación al trabajo es un posible factor causante del abandono de la lactancia materna en las madres evaluadas, ya que 36\% se incorporó inmediatamente después del parto y 30\% se reincorporó entre el primer y el tercer mes después del parto.13,14

Desde 2003 Navarro y colaboradores describieron que a pesar de que las madres se encuentran orientadas y conocen los beneficios de lactar, la mujer al incorporarse a sus actividades laborales, abandonan la lactancia materna por la ausencia de áreas apropiadas para la lactancia, se demostró la necesidad de intervenir en su área de trabajo para crear grupos de apoyo en el interior de sus centros laborales que permitan la extracción y almacenamiento de la leche materna, el cumplimiento por parte del empleador debe considerar a las madres desde el postparto para contar con esa áreas de seguridad, respeto y derecho. Por ende, todas las madres deben estar informadas y orientadas en sus derechos para poder exigir esto a su empleador..$^{15}$

El presente artículo se presenta como el primero de su clase en considerar a la población sudcaliforniana con respecto a los factores maternos asociados al abandonar lactancia materna exclusiva. La limitación es que al ser un estudio observacional puede tener bajas asociaciones.

\section{CONCLUSIÓN}

Los factores maternos asociados al abandono de la LME que influyeron en este estudio fueron inicio de actividades de la madre, enfermedad del lactante, que el niño no queda satisfecho, queda con hambre.

\section{AGRADECIMIENTOS}

Al personal del Área de Consulta Externa de Medicina Familiar del Hospital General de Zona con 
Medicina Familiar No 1. «Dr. Enrique Von Borstel Labastida», de La Paz, Baja California Sur, México.

\section{BibLIOGRAFÍA}

1. Oliver RA, Richart MM, Cabrero GJ, Pérez HS, Laguna NG, Flores AJ. Factores asociados al abandono de la lactancia materna durante los primeros 6 meses de vida. Rev Latino AM, Enfermagem, 2010; 18 (3): 80-87.

2. UNICEF. La lactancia materna es la forma más económica y eficaz de la historia para salvar vidas; únete por la niñez. México, D.F., 1 de agosto de 2013, comunicado de prensa, pp. 1-2.

3. Buenaño C, Chila I. Factores psicosociales que influyen en el abandono de la lactancia materna en madres adolescentes. [Tesis] Universidad Estatal de Milagro Facultad Ciencias de la Salud Milagro, Ecuador, mayo 2019.

4. Campiño-Valderrama SM, Duque PM. Lactancia materna: factores que propician su abandono. Arch Med (Manizales). 2019; 19 (2): 331-341.

5. Vázquez CL, Estany CA, Anido RM, Conde VM, Folgosa RM, Conzález VM et al. Efectividad de un taller formativo en lactancia materna exclusiva. Metas Enferm May. 2018; 21 (4): $75-21$.

6. Acosta SM, De la Rosa FJ. Causas que determinan la interrupción de la lactancia materna exclusiva en los barrios Santa Cruz y Propicia I en Esmeraldas, Ecuador. Rev Arch Med Camagüey. 2018; 22 (4): 434-444.

7. Rivera SC, Espinoza VH, Velasco PI, Suarez FR. Factores socioculturales asociados a la interrupción de la lactancia materna exclusiva en madres con niños menores de 6 meses. Revista Científica Mundo 56 Investigación y el Conocimiento. 2018; 2 (7): 3-25. ISSN: 2588-073X.

8. González HA, Martínez RC, Lorido C, Cubero S, Maynar MM, Gil MM. Situación actual de la lactancia materna en una zona de salud urbana y factores que la condicionan. Nure Inv. 2018; 15 (93): 1-14.

9. Prado CT, Sermeño PC, Chero PV. Determinantes sociales del abandono de lactancia materna exclusiva en niños a término menores de 6 meses en un Centro de Salud en San Juan de Lurigancho, 2017. Ágora Rev Cient. 2018; 05 (01): 1-6.

10. Martínez L, Hermosilla M. Razones de abandono de lactancia materna en madres con hijos menores de 2 años. Mem Inst Investig Cienc Salud. 2017; 15 (2): 73-78.

11. Reyes CA, Pereira DA, Pineda FM, Alcivar AD. Apego a la lactancia materna y factores que influyen en el abandono de la misma en la medicina privada. Acta Med. 2019; 17 (2):107-114.

12. Mínguez SM, Rodríguez SM, Burgos H. Prevalencia de lactancia materna en España. Revista Enfermería CyL. 2019; 11 (1): 12-15.

13. Cardona CA, Castaño CJ, Vallejo CS, Vargas MJ. Razones de abandono de la lactancia materna en madres de niños de jardines infantiles, Manizales, Colombia, 2015, Universidad de Manizales Facultad de Ciencias de la Salud Grupo de Investigación Médica. Manizales, 2015; 1-17.

14. Macay MR, Salas JL, Velez AA, Chavez MF. Abandono de la lactancia materna en las madres del Centro de Salud los huerfanitos. Revista Científica de Investigación Actualización del Mundo de las Ciencias. 2017; 14):390-402.

15. Navarro EM, Duque LM, Trejo PJ. Factores que influyen en el abandono temprano de la lactancia por mujeres trabajadoras. Salud Pública de México. 2003; 45 (4): 276-284. 